\title{
Raman4Clinics: the prospects of Raman-based methods for clinical application
}

\author{
Christoph Krafft ${ }^{1}$ Jürgen Popp ${ }^{1,2}$
}

Published online: 11 September 2015

(C) Springer-Verlag Berlin Heidelberg 2015

Cells and tissues are characterized by a specific biochemical composition and molecular structure. In a similar way, each pathology or cellular abnormality is accompanied by biochemical and molecular changes. Optical and spectroscopic techniques that correlate the biochemical composition, molecular structure, and their variations with the diagnosis would provide powerful clinical tools. The development of new optical methods for clinical diagnosis presents a challenge for natural scientists, including chemists, physicists, and biologists. In general, light-matter interaction is based on scattering, absorption, and emission of radiation. A variety of optical techniques have been suggested, including confocal fluorescence microscopy, photoacoustic imaging, infrared spectroscopy, optical coherence tomography, confocal reflection, two-photon excited fluorescence, and second-harmonic generation, to name a few. Many proof-ofprinciple reports have been published within the last few years, but only a few applications have been translated into the clinics. In particular, this holds true for Raman-based technology.

The physical mechanism behind Raman spectroscopy is inelastic light scattering of monochromatic laser radiation, which was experimentally discovered in the late 1920s. In the past decades, Raman spectroscopy has evolved as a versatile bioanalytical tool enabling label-free and chemically and

Published in the topical collection Raman4Clinics with guest editors Jürgen Popp and Christoph Krafft.

Christoph Krafft

christoph.krafft@ipht-jena.de

1 Leibniz-Institut für Photonische Technologien, Albert-Einstein-Str. 9, 07745 Jena, Germany

2 Institute of Physical Chemistry and Abbe Center of Photonics, Friedrich-Schiller University Jena, Helmholtzweg 4, 07743 Jena, Germany spatially resolved assessment of cells and tissue at the molecular level. All biomolecules, including proteins, lipids, nucleic acids, carbohydrates, and metabolites, are simultaneously probed within complex matrices without preparation, even under in-vivo conditions. This information provides a specific fingerprint and can be obtained at a variety of excitation wavelengths and intensities that are non-destructive to living organisms. Furthermore, implementation as a microscopic technique is capable of imaging molecular species at submicrometer resolution. Combination with fiber-optic probes enables minimally invasive application during endoscopy. The limitations of Raman spectroscopy regarding speed and throughput, caused by low signal intensities from most biological specimens, can be overcome by enhancement effects - surface-enhanced Raman scattering, resonance scattering, and coherent Raman scattering — or combination with other optical modalities.

Many research groups are currently active in the vibrant field of clinical Raman spectroscopy. The authors of the collection of papers in this topical issue come from Italy, Singapore, Canada, Ireland, France, United Kingdom, and Germany, which demonstrates the worldwide relevance of this research topic. The invited papers represent the five workgroups of the European COST action Raman4Clinics: biofluids, microbial pathogens, cytopathology, histopathology, and fiber-optic probes. The Raman4Clinics network was launched in 2014 to create a platform for scientific communication, exchange, and collaboration. Novel technology portfolios for clinical diagnostics are expected to emerge, to the benefit of patients and the economy.

The collection of papers in this topical issue will give recent examples of instrumental developments and biomedical applications in the context of Raman spectroscopy. The diagnosis of biofluids by variant surface-enhanced Raman scattering is reviewed. The procedure for isolation of bacteria from sputum samples is described for Raman spectroscopic analysis. The interaction of the antibiotic vancomycin with bacteria 
is identified by Raman spectroscopy. Cancer therapy is invitro predicted using Raman spectral imaging. Raman spectroscopy is reviewed for screening and diagnosis of cervical cancer. Raman spectroscopy under real-time conditions enables automatic skin-cancer detection. In-vivo Raman microspectroscopy of human skin correlates between spectral and biometric measurements during aging. A fiber-optic Raman probe within a hypodermic needle is characterized. Fiberoptic Raman spectroscopy is combined with fluorescencelifetime imaging as a complementary technique for prescreening. Endoscope-based beveled and volume fiberoptic Raman probes are compared for diagnosis of gastric dysplasia.

As guest editors of this special collection, we hope that the readers of these papers will be inspired regarding how their own research can contribute to overcoming the challenges of optical and spectroscopic clinical diagnosis. We thank all authors for submitting their interesting contributions, the referees for their reviews with critical but also constructive comments, and the editorial team of Analytical and Bioanalytical Chemistry for professional assistance.

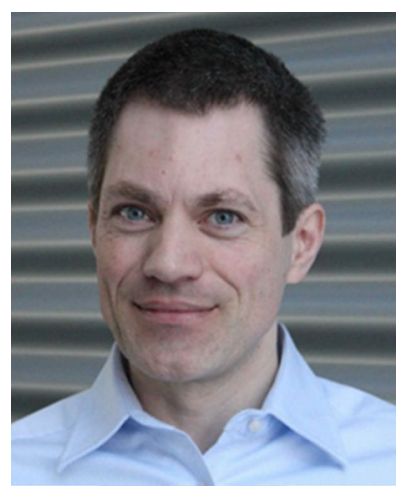

Christoph Krafft studied physics at the University Oldenburg. He worked as a $\mathrm{PhD}$ student at the Max Delbrück Center for Molecular Medicine and obtained his $\mathrm{PhD}$ degree in biophysics from the Humboldt University, Berlin. He joined the labs of George Thomas Jr. at University of Missouri, Kansas City (USA), and Valter Sergo at University Trieste (Italy) for postdoctoral work. As a leader of a junior research group at the University of Technology, Dresden, that was funded by the Volkswagen Foundation, he finished his habilitation in analytical chemistry in 2007. Since 2008 he has been working at the Leibniz Institute of Photonic Technology. His research interests are Raman and infrared spectroscopy of biomolecules, cells, and tissues, with a focus on microscopy, imaging, fiber-optic probes, and chemometrics for biomedical, clinical, and diagnostic applications. Since 1997 he has published more than 100 papers and book chapters in the field of biophotonics.

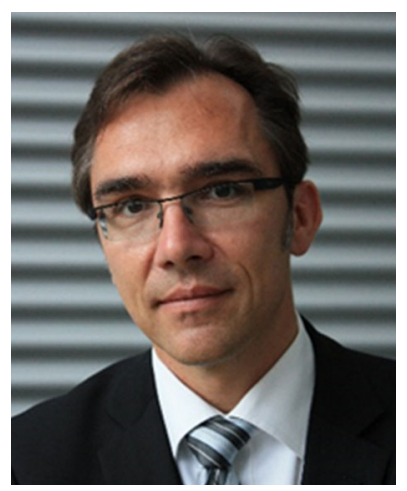

Jürgen Popp studied chemistry at the universities of Erlangen and Würzburg, Germany. After his $\mathrm{PhD}$ in Chemistry, he joined Yale University for postdoctoral work. He subsequently returned to Würzburg University where he finished his habilitation in 2002. Since 2002, he has held a chair for Physical Chemistry at the Friedrich-Schiller University, Jena. Furthermore, he has been the Scientific Director of the Leibniz Institute of Photonic Technology, Jena, since 2006. His research interests are mainly concerned with biophotonics. In particular, the development and application of innovative Raman techniques for biomedical diagnostics and environmental and food analysis is at the heart of his interests. He is a fellow of SAS and SPIE. In 2012 he received an honorary doctor's degree at Babes-Bolyai University Cluj-Napoca, Romania, in 2013 he received the Robert-Kellner Lecture Award, and in 2016 he will receive the Pittsburgh Spectroscopy Award. 